\title{
Human-computer super-intelligence
}

\author{
Alexander A. Antonov ${ }^{1 *}$ \\ ${ }^{1}$ Research Center of Information Technologies "Telannet Electronics" of the IAOI \\ * Correspondence author: tel/fax (380) 444243587; Email: telan@bk.ru
}

\begin{abstract}
The concept of human intelligence includes several types of intellectual activity - rational thinking, emotional thinking, unconscious thinking, intuitive thinking, and automatic control of the biological systems. Rational thinking refers to the cognition of the phenomena and laws of the animate and the inanimate nature. It is realized through the processing of mostly visual images and is therefore limited to the low-factor (not more than three-factor) laws of nature. Here belong, for instance, the laws of physics cognized by the mankind. All other types of human thinking are multiple-factor. The concept of artificial intelligence refers to computer simulation of the human low-factor rational thinking. This is why artificial intelligence cannot serve as the basis for 'computer super-intelligence'. Contrary to computer super-intelligence, the 'human-computer super-intelligence' can solve these tasks. Human-computer super-intelligence will allow developing super-knowledge on significant multiple-factor processes - medical-biological, scientific and technical, financial and economic, organizational, etc.
\end{abstract}

Keywords: mind and brain, human intelligence, human thinking, human knowledge, artificial intelligence, technological singularity, super-intelligence, super-knowledge.

\section{INTRODUCTION}

Ever since computers first appeared, people naturally wanted to turn them into intellectual assistants and partners of people. This is how the problem of artificial intelligence arose.

In order to turn computers into such intelligent devices, people decided first to 'teach' them (just like people) to talk, understand human speech, process visual images, position themselves in space, move, etc., i.e., basically those skills children naturally learn in their first year. And only later on it was expected to 'teach' computers to solve the most complicated, the so called intellectually demanding, tasks.

But what a child successfully learns in their first year, computer engineers failed to teach computers in almost fifty years. Therefore, a question arises as to what was wrong in the initial problem definition, and what the correct way to set the problem is.

Human intelligence: At present, there is no single conventional definition of intelligence, be it human or computer (Miles, 1957; Eisenk, 1995; Suvorov, 1999). Sometimes, using the terminology of physics, intelligence is referred to as 'the black box'.

Thus, we shall stick to the concept according to which human intelligence is an information system consisting of several various subsystems, or types of thinking. This concept was shared by Plato (Ambuel,
2007), Aristotle (Avraamova, 1970), Saint Albertus Magnus (Magnus, 2002) and many others. However, none of them, or anyone else, discussing their own conceptions of human intelligence and the meaning of the respective types of thinking, used the concepts of 'human low-factor thinking' and 'human multiplefactor thinking', the meaning of which is discussed below.

In accordance with the chosen concept, we shall distinguish the following types of human thinking, the definition of which in literature only sometimes and partially coincides with the definitions given below.

Rational thinking (Jung, 1971) serves to acquire new knowledge. It is also referred to as scientific, analytical, logical, abstract thinking. It is based mostly on the processing of visual images (which cannot be more than three-dimensional), and, due to this circumstance, is low-factor. Thus, rational thinking allows perceiving laws of nature in terms of functions having not more than three variables, and objects having not more than three dimensions. To make sure this is really so, try to imagine, for instance, a four-dimensional cube. Also, rational thinking is analog thinking, i.e., laws of nature perceived by it are continuous. Here belong, for instance, physical laws cognized by the mankind.

Emotional thinking (Mayer, 1990; Soros, 1994) (sometimes referred to as sensory perception) also 
serves to obtain new knowledge referring to certain particular, mostly nonrecurring and non-scientific, situations. Emotional thinking is multiple-factor, i.e., it reveals the relations referring to these situations, for more than three factors. However, as multiple-factor natural laws revealed by the human emotional thinking cannot be fully perceived by rational thinking, the results of this thinking are perceived in the simplified form as discrete, for example, in the form 'I like it'/ 'I don't like it'.

The unconscious thinking is far less investigated than the previous two types of thinking (Jung, 2002). Even at present it is barely being researched within scientific psychology. Therefore, let us give our own interpretation of this type of thinking, which, in our opinion, is the most important of all.

The starting point of our discussion is the phenomenon of sleep, the existence of which has not yet been given a complete explanation. The only things we know about it are as follows:

- Not only people sleep, but other living beings, too;

- All of them, just like computers, are in addition information machines, as they have a processor, memory and data-input devices;

- Human dreams are unique combinations of the events ever experienced by a person. This is why men (if they are not obstetricians) never dream of delivering a baby.

It is also known that:

- When a human is awake, their processor, memory and data input devices (sense organs) are working.

- When a human is asleep, their processor and memory work, but their data input devices (sense organs) are off.

Why do we need this? In order to comprehend the problem, let us compare the following situations:

- When a human is awake, they act, and these are life supporting activities without which a human cannot exist.

- When a human is asleep, they are inactive, this is why we can get the impression that we do not need sleep, and that sleep is even harmful as, for instance, in sleep a human is less protected.

However, if Nature uses the sleep mode, it means Nature has some strong reasons for it. This is why we have to understand them. This circumstance was first pointed to by Saint Albertus Magnus. As the only difference of the sleep mode from the wake mode lies in ceasing any activity, and, therefore, turning off data input devices, it means that in the sleep mode it is necessary for the processor and memory to perform joint activity without any disturbing factors. This activity can be only a different type of thinking; moreover, this type of thinking is so virtually important and time-consuming that it is impossible to refuse from it.

Thus, a human thinks in their sleep, as well. And this is the most important function of sleep. In sleep people process information inputted in their memory when they were awake. It was Plato who wrote that the outside world is the basis for human intelligence.

But under the guidance of rational thinking humans study, write scientific works, make inventions, compose music, write literary works, make business only in the wake mode. Is this not the most important type of thinking? There can be only one answer - all types of thinking are essential, and in this sense all of them are important, as only the combination of all these types of thinking allowed a human being to survive and to develop.

Let us remember here that rational thinking in the wake mode is low-factor. This is why it allows a human to think relatively fast, which is necessary for most activities.

In sleep, the slower, but more productive multiplefactor thinking starts to work. As this is a much more complicated type of thinking, it requires much more time and memory. This is why in the sleep mode the data input devices (i.e. the sense organs) turn off in order not to hamper the process of multiple-factor thinking.

Therefore, the saying 'if you have a problem, sleep on it' is absolutely correct, as processing (complete comprehension) of information stored in memory during the day is completed by next morning.

Moreover, a human very often uses the results of the multiple-factor unconscious thinking without being able to explain it and without even being aware of it. For instance, this is why someone who is sick has lower fever in the morning than at night. That is, a human body uses the results of the unconscious thinking for self-treatment, as well.

As for dreams, they are explained by the use in the process of the unconscious multiple-factor thinking of those areas of the brain which are responsible for the process of vision when a human is awake. Therefore dreams can indeed give an insight into the process of the unconscious multiple-factor thinking. But we need 
to take into account that dreams are only a mere reflection of some low-factor fragments of a multiplefactor process.

Dreams also prove that a human thinks with visual, but not acoustic or any other images, as in sleep a human only sees something, but does not hear anything and does not perceive any other sensations, although other areas of the brain, which in the wake mode are responsible for the sense of smell, tactile sense, and hearing, are also involved in the process of the unconscious thinking.

When a human sometimes needs the results of the multiple-factor unconscious thinking to have an effect on their life in the wake mode, these results are communicated to them without any explanations in the form of concepts available for the rational and the emotional thinking (Kahneman, 2002), i.e. in the form of recommendations on further actions. This information is often referred to as insight (e.g. scientific), intuition, inspiration, revelation (e.g. in art), inner voice (e.g. in business), apprehension. This information should be paid the utmost attention.

The ability of intuitive thinking should be developed. Presumably, special equipment could be used for this purpose in the interactive mode (for instance, devices designed similar to a lie detector).

Finally, human thinking is used for automatic control of a human's numerous biological systems - blood circulatory system, digestive system, immune system, respiratory system, etc.

Apart from the above mentioned systems the existence of which is genetically defined, there are systems of automatic control acquired by a human in the process of their activity - for instance, the system of supporting the balance when walking.

Thus, human intelligence is realized by nature as a system of several subsystems of thinking, which function in a different way and all together allow solving the task of survival of a human as a biological species (Anokhin, 1971). Human low-factor rational thinking which allows making fast decisions on tactical tasks is supplemented with a set of other types of human multiple-factor thinking providing for slower strategic thinking. But people usually are not aware of the existence of their multiple-factor thinking and believe that all their thinking is just low-factor rational thinking. However, the subsystem of human rational thinking does not allow comprehending multiple-factor laws of nature due to their far greater complexity compared to low-factor natural laws.
The use of mathematical statistics is an attempt to try to find a way of comprehending multiple-factor natural phenomena with low-factor human rational thinking. However, mathematical statistics gives rather scarce knowledge. For instance, despite several dozens of statistical estimates of economic activity, people do not completely understand these processes, and their battle with economic crises is not very successful.

Knowledge can be conscious, i.e. can be set out in words, and unconscious, i.e. impossible to define verbally.

Conscious knowledge is knowledge obtained due to low-factor rational thinking. People get conscious knowledge during their course of study at schools and during their self-use of rational thinking, e.g. during scientific research or labor activity.

Unconscious knowledge (sometimes also referred to as implicit) is knowledge obtained using the subsystems of multiple-factor thinking. Unconscious knowledge - how to talk, to recognize one's parents and other objects of the outside world, to walk without losing one's balance, etc., children get during their first year of life. Sometimes this type of knowledge can be obtained by adults - for instance, the skills of driving a car, riding a bike, skating, swimming, etc. This confirms the possibility of teaching with the use of the unconscious thinking in any age.

It is easy to note that the unconscious knowledge is more convenient for practical use - for instance, it is easier for people to speak their native language leaned in their early childhood through the subsystems of multiple-factor thinking, than a foreign language learned at school using the low-factor rational thinking. Thus, we can assume that the most efficient methods of teaching are those based on the use of multiple-factor unconscious thinking, and these methods should be used as widely as possible.

Human knowledge bases store both the conscious and the unconscious knowledge. But it is virtually impossible to study the unconscious multiple-factor knowledge using human low-factor rational thinking.

Artificial intelligence: Soon after computers were created due to the work of many prominent scientists and engineers (Rojas, 2001), people realized the need of using them to solve more intellectual tasks than mathematical calculations (be it even the most complicated ones).

Back in 1950, the article (Turing, 1950) was published; it was afterwards reprinted many times, 
including under the title 'Can the Machine Think?' This article was the starting point for the problem of artificial intelligence. However, the term 'artificial intelligence' (AI) was offered later, in 1956 (McCarthy, 1959).

The problem of artificial intelligence was very popular and extensively studied by electronic engineers, software developers, mathematicians, neurophysiologists, philosophers, psychologists, linguists, and others. Naturally, their interpretations of the problem, means of solving it and objectives of research differed greatly.

However, (Feigenbaum et al., 1989) suggested a definition which has, probably, the least objections: artificial intelligence is a sphere of computer science engaged in development of intellectual computer systems, i.e. systems having the capabilities traditionally associated with human thinking understanding of speech, learning, reasoning, problem solving, etc.

There are two largest schools researching the problem of artificial intelligence using two different approaches (Copeland, 2000), namely:

- the top-down Al or cybernetic approach, and

- the bottom-up $\mathrm{Al}$ or neurophysiologic approach.

We shall use the latter pair of terms - the cybernetic approach and the neurophysiologic approach, as they more accurately reflect the essence of the respective research schools.

Supporters of the neurophysiologic approach believe that first we need to study the human brain in order to understand the ways it solves various intellectual tasks, and what human intelligence actually is, and only after that we can develop devices emulating and improving the human brain. This approach makes sense. However, its drawback is that knowledge of neurophysiologic processes is not enough to understand the work of the human brain.

Adherents of the cybernetic approach quite logically believe that technical devices improving the work of the human brain can and must be developed without emulating its work. They refer to the fact that, for instance, ground vehicles created by engineers use wheels, and do not emulate the movement of terrestrial living creatures using legs. And aircraft use motionless wings or do not use wings at all, and do not emulate the flight of birds and insects having flapping wings.
A gyroscope also does not emulate the vestibular apparatus of a human.

Let us give another example. For a human, the simplest mathematical operations are those of addition, subtraction, multiplication and division, and mathematical operations of differentiation and integration are more complex. In analog radioelectronics, mathematical operations of differentiation, integration, addition and subtraction are easiest to implement, whereas mathematical operations of multiplication and division are more complex.

In other words, the criteria of simple and complicated in nature and in human machine civilization differ greatly. Thus, the principles of operation of technical devices will inevitably differ from those of living beings.

Nevertheless, at the initial stage of researching the Al problem, adherents of both approaches set basically the same task of emulating the process of human learning, i.e. developing the software allowing computers to understand human speech, to talk to people, to process visual images, to position themselves in space and to move, that is, the skills children acquire in their first year. Evidently, researches then believed that these tasks are the simplest in the Al problem. This is why development of software allowing solving more intellectual, and therefore more complicated tasks, from the human point of view, was supposed to be done later.

However, researchers somehow forgot that ever since computers were created, they were successful in solving the tasks of the so called higher mathematics, which people learn to solve in their adulthood. That is, the task which seemed complicated for people turned out to be very simple for computers.

And vice versa, the above listed simplest human tasks initially set for GOFAl (Good Old-Fashioned Artificial Intelligence), have not yet been solved (Newell and Simon, 1972; Dreyfus, 1972; Haugeland, 1989; Luger, 2004; Kuklin, 2004), and, moreover, will not be solved any time soon.

Finally, the tasks initially set for the Al have been corrected (Munakata, 2001; Hoya, 2005; Minsky, 2006; Goertzel and Pennachin, 2007; Russell and Norvig, 2009) and are now being successfully solved. These tasks include development of real time expert systems, or dynamic expert systems (Ali et al., 1997; Jackson, 1999; Giarratano and Riley, 2004), 
investigation of neural networks (Parks et al., 1998; Borovikov, 2008), tasks in the sphere of robotics (Bräunl, 2006; Manseur, 2006) and others.

However, the question why the tasks initially set for GOFAl remained unsolved is still unanswered. The answer to it is rather simple. The point is that when the tasks were initially set researchers did not take into account the above mentioned peculiarities of human multiple-factor thinking. Thus, extremely complicated multiple-factor tasks were supposed to have been solved by the simplified human low-factor thinking emulated by the computer.

Let us remember here that low-factor human thinking has been intended by Nature not to perform scientific research, but to solve the tasks of survival. And the unconscious multiple-factor human thinking, which is able to solve the most complicated tasks, including scientific, is incomprehensible for the low-factor rational human thinking.

Thus, a question arises: why did Nature create human thinking in such an illogical way? Why does human mind have to solve complicated multiplefactor tasks in the first days and months of a human life, and use the more simple low-factor rational thinking in the adulthood?

The answer is obvious - in order to survive. It turns out that multiple-factor childish unconscious thinking is the most efficient teacher. It is due to this thinking that in the early days of life a human baby is able to quickly acquire the knowledge and skills necessary for survival. This circumstance accounts, in particular, for the 'Mowgli phenomenon'. As for the low-factor rational thinking of adults, it merely supports these survival skills later on.

Technological singularity: However, despite huge difficulties in practical implementation of GOFAI, there is one more opinion on the computer development prospects - it is suggested that rapid improvement of computers will lead to the emergence of computer civilization on our planet (Moravec, 1988; 1998; Vinge, 1993; Kurzweil, 2005). This scenario of Al development came to be known as 'technological singularity', which is defined as hypothetical explosive acceleration of the scientific and technical progress having unpredictable results. Moreover, there are several scenarios of transition to the computer civilization:

- Super-intelligence will be the result of improvement of computers;
- Self-developing information networks (e.g. the Internet) can develop into superintelligence;

- Intelligence of computer users will develop to the extent of super-intelligence due to improvement of the human-computer interface;

- Nanotechnologies and bioengineering will improve the biological nature of the mankind to such an extent (Drexler, 1986; 1992; Merkle, 1993) that humans will become super-intelligent.

The definition of super-intelligence was first suggested in (Bostrom, 2006), however, the question as to how super-intelligence can be implemented in practice remained open. Nevertheless, it is assumed that super-intelligence will certainly be realized sooner (by 2016 or 2018) or later (by 2030), irrespective of the willingness or unwillingness of people.

And this circumstance raises serious concerns (Nelson, 2000; Yudkowsky, 2006). Paper (Good, 2006) even argues that the first super-intelligent computer will be the last invention of the mankind.

\section{Human-computer super-intelligence:}

The concerns mentioned in the previous section are raised by:

- the fact that computer super-intelligence is very likely to develop;

- absence of any specific and persuasive positive scenarios of relationship development between the computer and human civilizations;

- and even absence of any guarantees of further existence of the human civilization after the emergence of computer superintelligence.

However, the issue of the greatest concern is that basically we are dealing with complete misunderstanding of the computer super-intelligence phenomenon and its frightening differences from human intelligence. Indeed, where do these differences lie?

- In the amount of information?

- In the rate and algorithms of information processing?

- In any new principles of presenting information?

- In the new supercomputer ethics? Or in anything else? 
As there are no answers, it becomes clear that we have lost control over the development of the situation.

The validity of the above can be easily verified if we try to answer the following questions:

- In what way will the new super-knowledge differ from human knowledge?

- Is a man actually able to comprehend this knowledge if, supposedly, a friendly computer super-intelligence (or any other friendly civilization) would be willing to share its super-knowledge with the mankind?

- And what is actually super-knowledge?

If the new biotechnologies and nanotechnologies are somehow able to improve the information characteristics of humans, the question is which ones and in what way? If the new human-computer interfaces are created, the question is how and in what way will they allow people to comprehend super-knowledge?

There are many questions. However, the destiny of human civilization depends on the answers. Evidently, it is necessary to revise its fundamental abilities to survive and, respectively, correct the basics of its existence. Unfortunately, natural selection and altruism are incompatible, with regard to our human civilization, as well.

So, does the human civilization have no way out, and is it doomed?

Before answering this question, let us figure out how exactly super-intelligence and super-knowledge differ from the existing human intelligence and existing human knowledge, and what it actually is.

In our opinion:

- super-knowledge is multiple-factor knowledge, i.e. knowledge of multiple-factor objects and processes, including, for instance, biological nature of a man, the mechanisms of human illnesses, the mechanism of ageing, the global and regional economy, the weather and the environment, the processes of mineral deposits formation, state and corporate governance, and even processes still continuing in the destroyed reactors of the Chernobyl nuclear power plant;

- super-intelligence is the ability to comprehend the above mentioned superknowledge and use it in the best possible way.
Thus, the prospects of further existence of human civilization depend only on the ability or inability of the mankind to comprehend and use significantly multiple-factor information.

Fortunately, as we have mentioned above, human intelligence is based on multiple-factor thinking. Therefore, we can assume that a human is potentially super-intelligent, i.e. super-intelligence can possibly be based on human intelligence. In order to fulfill this possibility, super-intelligence should be based not only on human thinking, but on computer resources, as well, i.e. super-intelligence should be humancomputer.

Speaking about the computer base, human-computer super-intelligence is possible on the basis of either supercomputers or computer networks. Therefore, we will consider these two implementations in more detail.

For a more detailed discussion of the problems of super-intelligence and super-knowledge, the variant of its implementation of the basis of supercomputers is more convenient as it allows omitting many technical peculiarities insignificant at the beginning.

Indeed, let us suppose for simplicity that we already have a supercomputer with the best possible features we can imagine. Let us also assume that it stores all the conscious knowledge accumulated by the mankind. We shall not discuss when, how, on what basis and at what cost these results can be achieved. This is not relevant right now.

What is relevant is the answer to the question how the human-computer super-intelligence will be able to solve multiple-factor tasks.

Without trying to embrace the un-embraceable, hereinafter we shall discuss only the solution of scientific multiple-factor tasks, as they are probably of the greatest practical interest and are most understandable.

Indeed, simplistically the process of scientific intellectual activity (or scientific intellectually demanding activity) can always be clearly divided into two stages which are substantially different:

- detection of factors which have a significant influence on the process under investigation, and screening of irrelevant or insignificant factors;

- determination of mathematical (or other cause-and-effect) relation between the revealed significant factors. 
Meanwhile, the process under investigation may be both low-factor and multiple-factor. It depends on the problem statement, financing, the researcher and their talent, competitive environment, and many other circumstances.

In terms of solving low-factor problems, the most difficult for people in the process of creative thinking is the first stage, i.e. detection of significant factors influencing the results of the process under investigation. Finding mathematical dependence between the revealed significant factors (e.g. based on the results of experimental studies) in this case is relatively a much simpler task.

In terms of solving multiple-factor problems, the complexity of performing the tasks corresponding to both stages increases to such an extent that solving such a problem becomes virtually impossible for a human. This is why in physics, for instance, there are no laws described with functions of over ten variables, although such laws certainly exist in nature.

But for a computer having the relevant hardware and software, the number of factors is rather a quantitative than a qualitative difference. This is why a computer, together with its user, is able to successfully analyze multiple-factor processes.

However, without human intelligence such intellectually demanding work of a computer is most likely impossible to be implemented in the foreseeable (quite distant) future. And this shouldn't be done in any case! In order to make sure such a scenario is prevented, special measures will have to be taken (similar to, e.g., Isaac Asimov's robotics laws). In this case the emergence of purely computer civilization which does not cooperate with the human civilization will be prevented.

Just like we need different types of business, both small and large, we need various types of scientific research, both teamwork and individual. Science having no alternatives, in the same way as business lacking competitive environment, cannot develop. Creative work is always inherently individual. Only on condition of making the most favorable conditions for realization of the researchers' creative potential is it possible to develop the human-computer superintelligence.

This is why the network implementation of humancomputer super-intelligences would be most suitable for the majority of scientists, as it is more easily accessible. We are speaking about super- intelligences, but not about a single superintelligence. That is, human-computer superintelligences should mostly be implemented by users based on their PCs, and they should be numerous.

Consequently, national and international programs supporting economic and scientific development of the mankind, aimed at the development of superintelligence, should be able to provide for the best possible efficiency of individual scientific research. In order to do this, it is necessary:

- to make scientific competition as fair as possible by making infringement of copyright and false joint authorship impossible;

- to provide the conditions for most beneficial financing terms for efficient researchers, including the use of venture financing and other means of involving enterprisers' talent;

- to provide the researchers with unlimited possibilities for publishing the results of their investigations;

- to eliminate the 'suppression areas' of science not belonging to classified research, which cannot be discussed nowadays due to the fact that some problems of science have not been scientifically understandable for a long time (decades, centuries and even millenniums).

On the contrary, people should have complete and objective information on these unsolved problems. In particular, this information activity should be one of the top priorities of the global education system. Textbooks should contain not only the results of scientific research, but describe the thorny path leading to these discoveries, as well as the remaining questions and unresolved problems.

Apart from the above mentioned organizational and ethical conditions for the development of humancomputer super-intelligence, it is necessary to create certain technical prerequisites. In case of development of the human-computer superintelligence based on personal computers we cannot assume that the latter have the best possible technical features, as it was in the case of supercomputers. PCs are much cheaper and simpler devices, and we have to take this into account. Therefore, we should bear in mind that a personal computer used for solving super-intelligent tasks should have first of all the largest possible peripheral memory, as in order to determine the factors having significant influence on the process under investigation it is necessary to analyze a huge amount of source information. However, this 
peripheral memory will still have a smaller storage capacity than that of supercomputers, as superintelligence based on PCs will be used to solve less ambitious problems.

Nevertheless, it would be impossible to manually upload all the necessary source data to this peripheral memory. It will have to be connected to the new global information network operating in the mode of continuous computer broadcasting (Antonov, 2009).

The Internet would not be able to serve the purpose. First of all, it simply does not have the required information. And the search of those grains of information it does have takes a lot of time, as the Internet is highly littered with information which has little or no value and with information no one actually needs.

Secondly, the new information network should be able to guarantee full information security for its users, as otherwise an infected and modified by anyone at any time super-intelligence can become unpredictable and therefore extremely dangerous. Thus, the use of packet connection for the new information network is absolutely out of the question. Due to this, human-computer super-intelligence cannot be based on any type of the Internet.

Certainly, the necessary hardware and software also have to be developed for personal computers solving super-intelligence tasks.

And, finally, we do have to stress that in order to avoid irresponsible and criminal use of superintelligence, this activity should be strictly regulated and controlled.

\section{CONCLUSION}

6.1. A human intelligence is a complex information system consisting of several interacting subsystems, where the human rational thinking is low-factor, and other types of human thinking are multiple-factor.

6.2. Human rational thinking serves to quickly solve tactical aspects of the problem of survival, and multiple-factor subsystems of human thinking solve the most important strategic aspects of this problem.

6.3. The existing conscious human knowledge acquired with the help of human rational thinking is low-factor knowledge. Human knowledge obtained with the help of other subsystems of human thinking is multiple-factor and unconscious knowledge.

6.4. Presumably, some ways of improving human intelligence which have not yet been demanded by the evolution - the use of developed intuition, conscious use of the unconscious multiple-factor thinking, etc. - can be realized, including the use of the new hardware and software.

6.5. Human intelligence which developed during millions of years of biological evolution is the most perfect system in terms of its performance, and allows creating super-intelligence on its base.

6.6. In the foreseeable future any threats to the existence of human civilization from computer civilization within the concept of technological singularity can be eliminated.

6.7. The prospects of further existence of the human civilization are determined only by the ability or inability of people to comprehend and use multiplefactor information.

6.8. Rapid development of computer technology and adjacent information technologies allows assuming that human-computer intelligence will develop in the nearest decades, which will be able to respond to multiple-factor situations in the best possible way.

6.9. Human-computer super-intelligence can be realized both on the basis of supercomputers and computer networks consisting of personal computers.

6.10. However, in case of negligent, irresponsible or criminal use of the human-computer superintelligence, the latter can be very dangerous for the mankind.

6.11. The use of human-computer superintelligence will result in the all-round rapid development of the human civilization and its eventual development into the new human-computer civilization.

\section{REFERENCES}

Ali, M., Potter, D. \& Matthews, M. (1997). Industrial and Engineerin Applications of Artificial Intelligence and Expert Systems. London: Taylor \& Francis.

Ambuel, D. (2007). Image and Paradigm in Plato's Sophis. Las Vegas: Parmenides Publishing.

Anokhin, P. K. (1971). Philosophical aspects of the functional system theory. Problems of philosophy, 3, 55-60.

Antonov A.A. (2009). Safe Global/Regional Informational Network. European Journal of Scientific Research, 28 (1), 165-174.

Avraamova, M. A. (1970). Aristotle's doctrine on substance. Moscow State University.

Borovikov, V.P. (2008). Neural networks. Methodology and technologies of contemporary data analysis. Moscow: Hotline - Telecom

Bostrom, N. (2006). How Long Before Superintelligence? Linguistic and Philosophian Investigations, 5 (1), 11 - 30. 
Bräunl, T. (2006). Embedded Robotics: Mobile Robot Design and Application with Embedded Systems. (2nd ed). Berlin: Springer.

Copeland, J. (2000). What is Artificial Intelligence? The Turing Archive for History of Computing. University of San Francisko.

Drexler, K.E. (1986). Engines of Creation: The Coming Era of Nanotechnology. NY: Anchor Books.

Drexler, K.E. (1992). Nanosystems: Molecular Machinery, Manufacturing, and Computation. NY: John Wiley \& Sons, Inc.

Dreyfus, H. L. (1972). What Computers Can't Do: The Limits of Artificial Intelligence. NY: MIT Press.

Eisenk, H. Yu. (1995). The concept and definition of intelligence. Problems of psychology, 1, 111 - 129.

Feigenbaum, E.., Barr, A.. \& Cohen, P. (1989). The Handbook of Artificial Intelligence. (Vol. 4). Reading, MA: AddisonWesley.

Giarratano, J.C. \& Riley, G.D. (2004). Expert Systems: Principles and Programming. Course Technology. (4th ed). Boston, MA:. Thompson Course Technology Inc.

Good I.J. (1965). Speculations Concerning the First Ultraintelligent Machine. Advances in Computers, 6, $31-$ 88.

Goertzel, B. \& Pennachin, C. (2007). Artificial General Intelligence (Cognitive Technologies). NY: SpringerVerlag.

Haugeland, J. (1989). Artificial Intelligence: The Very Idea. Cambridge, MA: MIT Press.

Hoya, T. (2005). Artificial Mind System. NY: Springer-Verlag.

Jackson, P. (1999). Introduction to Expert Systems. (3rd Ed). Reading, MA: Addison-Wesley.

Jung, C. G. (1971). Psychological Types. Princeton University Press.

Jung, C. G. (1990). The Archetypes of the collective unconscious. Princeton University Press.

Kahneman, D. (2002). Nobel Prize Lecture: Maps of bounded rationality. A perspective on intuitive judgment and choice.

Kuklin V.M. (2004). Contamination with intelligence, or ways of creating artificial intelligence. Universities. (Vol. 4). Kharkov: Publisher Media-Group Okna.

Kurzweil, R. (2005). The Singularity Is Near: When Humans Transcend Biology. NY: Viking.

Luger, G.F. (2004). Artificial intelligence. Structures and Strategies for Complex Problem Solving. (5th Edition). Reading, MA: Addison Wesley.

Manseur, R. (2006). Robot Modelling \& Kinematics (Computer Engineering). Rockland, MA: Charles River Media.

Mayer, J.D., DiPaolo, M.T., \& Salovey, P. (1990). Perceiving affective content in ambiguous visual stimuli: $A$ component of emotional intelligence. Journal of Personality Assessment, 54, 772-781.

McCarthy, J. (1959). Programs with Common Sense. Proceedings of the Teedington Conference on the Mechanization of Thought Processes. London.

Merkle, R.C. (1993). Molecular Manufacturing: Adding Positional Control to Chemical Synthesis. Chemical Design Automation News, 8, 9 \& 10, 1-11.

Miles, T. R. (1957). On defining intelligence. The British Journal of Educational Psychology, 27 (3), 153 - 167.

Minsky, M. (2006). The Emotion Mashine: Commonsense Thinking, Artificial Intelligence, and Future of Human Mind. NY: Simon \& Schuster.

Moravec, H. (1988). Mind Children: The Future of Robot and Human Intelligence. Cambridge, MA: Harvard University Press.

Moravec, H. (1998). When will computer hardware match the human brain? Journal of Evolution and Technology, 1, 1 12.

Munakata, T. (2001). Fundamentals of the New Artificial Intelligence: Beyond Traditional Paradigms (Texts in Computer Science). Berlin:Springer.

Nelson, J.W. (2000). Why the future doesn't need us. Wired magazine, April, $1-11$.

Newell, A. \& Simon, H.A. (1972). Human problem solving. Englewood Cliffs: Prentice Hall.

Parks, R.W., Levine, D.S. \& Long, D.L. (1998). Fundamentals of Neural Network Modeling. Cambridge, MA: MIT Press.

Rojas, R. (2001). Encyclopedia of Computers and Computer History. Chicago: Fitzroy Dearborn Publishers.

Russell S. \& Norvig P. (2009). Artificial Intelligence: A Modern Approach. (3rd Ed). Englewood Cliffs, NJ: Prentice Hall.

Saint Albertus Magnus. (2002). On intelligence and the intelligible. Anthology of the Medieval thought. Theology and philosophy of the European Middle Ages. Publishing House of the Russian Christian Humanitarian Institute.

Soros, G. (1994). The Alchemy of Finance. Reading the Mind of the Market. NY: John Wiley \& Sons.

Suvorov, V. V. (1999). Intelligence is the axiom for psychic reality. Herald of Moscow State University, Psychology, 14 (3), $66-75$.

Turing, A. M. (1950). Computing Machinery and Intelligence. Mind, 59, $433-460$

Vinge, V. (1993). The coming technological singularity: How to survive in the post-human era. VISION-21 Symposium. NASA Lewis Research Center and the Ohio Aerospace Institute.

Yudkowsky, E.S. (2006). Artificial Intelligence as a Positive and Negative Factor in Global Risk. Palo Alta, CA: Singularity Institute for Artificial Intelligence. 